\title{
A case of rare stroke syndrome admitted in intensive care unit - Artery of Percheron infarction
}

\author{
K.Gunasekaran $^{1 *}$, S. Sivakumar ${ }^{2}$, K.Thiruvarutchelvan ${ }^{3}$ \\ ${ }^{\mathbf{1}}$ Assistant Professor, ${ }^{2}$ Professor and HOD, ${ }^{3}$ Professor, Dept. of Neurology, Government Mohan Kumaramangalam Medical College \\ Hospital, Salem, Tamil Nadu, India
}

*Corresponding Author: K.Gunasekaran

Email: drgunasekaranneuro@gmail.com

\begin{abstract}
Artery of Percheron is a rare anatomical variant of thalamic vascular supply. Stroke involving this vasculature is an uncommon event. Occlusion of this vessel produces a peculiar pattern in the form of bilateral thalamic infarcts with or without midbrain infarction. This results in variable clinical features ranging from somnolence or altered sensorium to coma in affected individuals, accompanied by characteristic neurological manifestations. Diagnosis of artery of Percheron infarction at the initial presentation may be difficult as it is infrequent and early neuroimaging studies may be negative. Here we report a patient, a case of AOP infarct whose MRI brain and clinical picture demonstrated the characteristic features.
\end{abstract}

Keywords: Artery of Percheron, Ischemic stroke, Thalamus, Magnetic resonance Imaging.

\section{Introduction}

Among the various posterior circulation stroke syndromes, artery of Percheron (AOP) infarction is a rare presentation. Occlusion of AOP results in characteristic neurological signs and symptoms as there is impairment of perfusion occurs in thalamus alone or thalamus with midbrain bilaterally. Among the cerebral ischemic infarcts, AOP occlusion constitutes $0.1 \%$ to $0.3 \%$ of cases. ${ }^{1}$ In ischemic infarct involving thalamus, AOP obstruction constitutes $22 \%$ to $35 \%$ of cases. ${ }^{2}$ Early diagnosis of this stroke may be difficult because it is an uncommon one and neuroimaging with computed tomography (CT) scan or magnetic resonance image (MRI) scan may not be contributory at initial stage. More over some other posterior circulation stroke syndromes may manifest similar features which will result in diagnostic dilemma. Here we describe a patient, who was admitted in our ICU with features of posterior circulation stroke in whom diagnosis of this rare condition was made out by characteristic MRI features.

\section{Case Report}

Mrs.V, a 61 years old woman, admitted in ICU of our hospital with complaints of drowsiness since the morning. Patient was apparently normal previous night, next day morning found to be drowsy and responding poorly to calls. Patient was a known hypertensive, irregularly on drugs and not a diabetic. There was no history of injury, fever, recurrent vomiting or seizure. There were no similar symptoms or any neurological illness in the past. On examination, her vital signs were: BP: 150/90 mmhg, PR: $88 /$ min. Respiration and temperature were normal. Examination of higher mental function: patient was confused, stuporous but arousable and going for sleepy state frequently. Word output was less with slurring of speech noted. There was no aggressive behaviour or hallucinations. Cranial nerves examination showed bilateral complete ptosis with bilateral external ophthalmoplegia. Except minimal abduction in left eye all other movements were absent. Oculocephalic reflex was absent. Vertical gaze was absent. Both sides pupils were $2.5 \mathrm{~mm}$, sluggishly reacting to light. Other cranial nerves were normal. Palatal movements were normal. Spinomotor system examination: patient was able to move all the four limbs, tone in limbs were normal and deep tendon reflexes were retained. Bilateral plantar reflexes were mute. Sensory system could not be tested. Cerebellar system: though the patient was able to walk with support, gait ataxia was noted. Other systems were clinically normal. With these clinical features, possible diagnosis of stroke involving the posterior circulation was made out and management was initiated with basic and supportive care. Her investigations were as follows: complete hemogram, renal function tests and blood glucose were within normal limits. ECG showed no significant changes. MRI brain T2/FLAIR (fluid-attenuated inversion recovery) sequences showed hyperintense signal in bilateral paramedian region of thalamus and rostral part of midbrain (Fig. 1, 2 \& 3) with diffusion restriction in same regions in DWI (diffusion - weighted images) (Fig. 4), which were in favour of acute infarct. Also the characteristic 'V' sign (Fig. 5, 6 \&7) was present. MR cerebral angiography (Fig. 8) and venography were unremarkable. Based on these clinical picture and neuroimaging findings diagnosis of AOP infarct was made and treated accordingly.

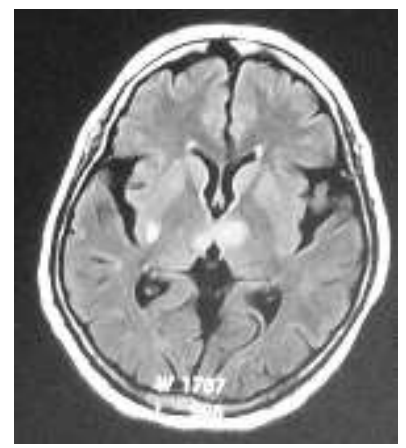

Fig. 1: T2 FLAIR axial view bilateral thalamic infarct 


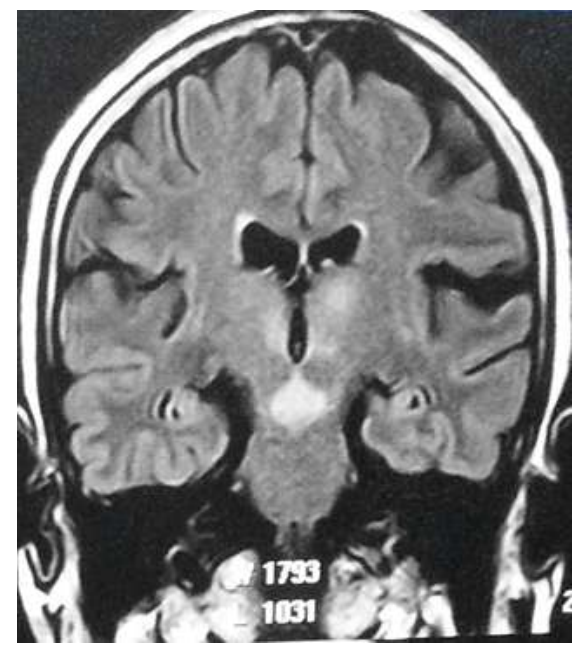

Fig. 2: T2 FLAIR coronal view showing infarct in thalamus and midbrain

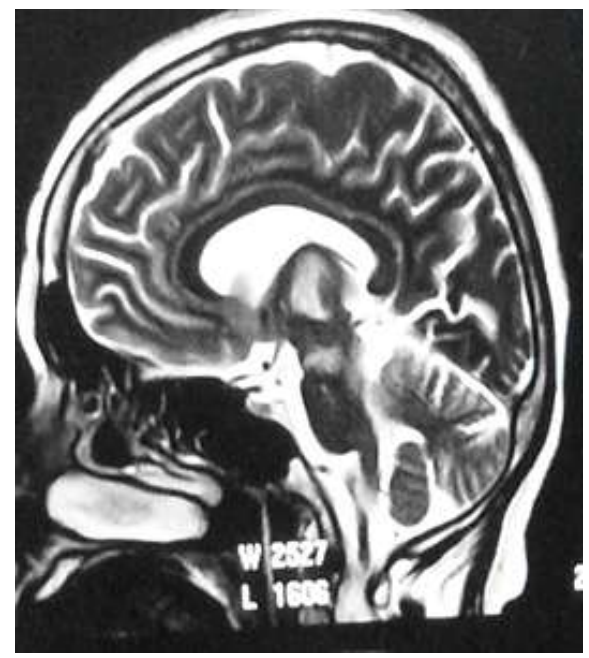

Fig. 3: T2 sagittal view showing infarct in thalamus and midbrain

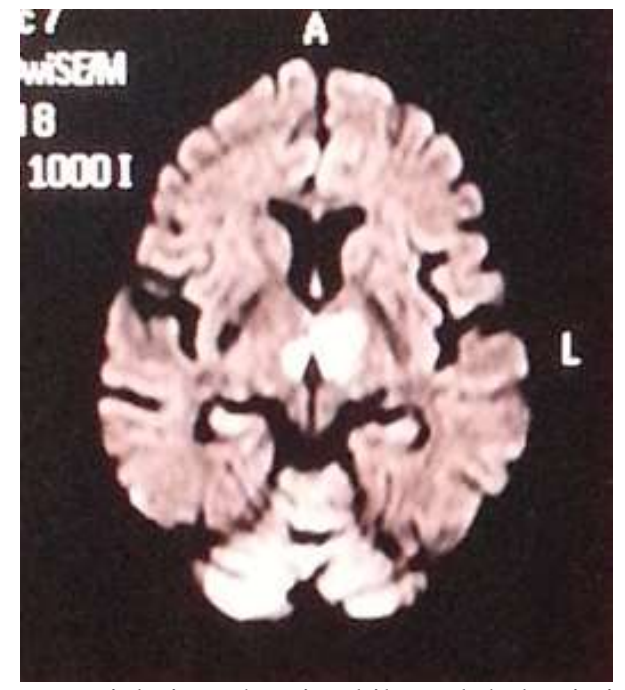

Fig. 4: DWI axial view showing bilateral thalamic infarct

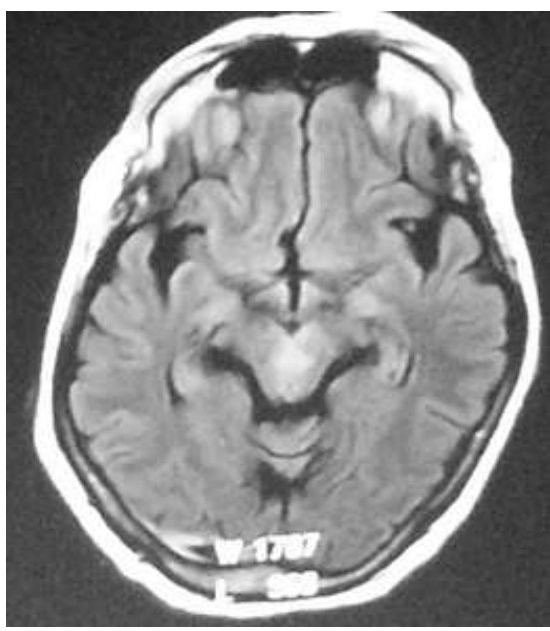

Fig. 5: T2 FLAIR axial view showing V sign in midbrain

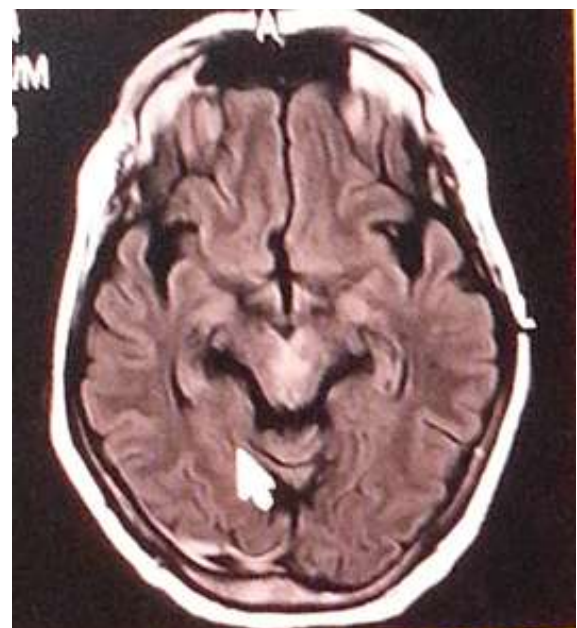

Fig. 6: T2 FLAIR axial view showing V sign in midbrain

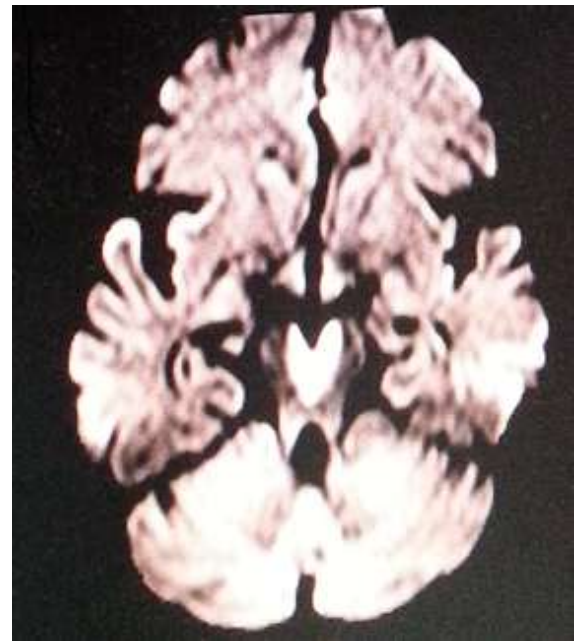

Fig. 7: DWI axial view showing V sign in midbrain 


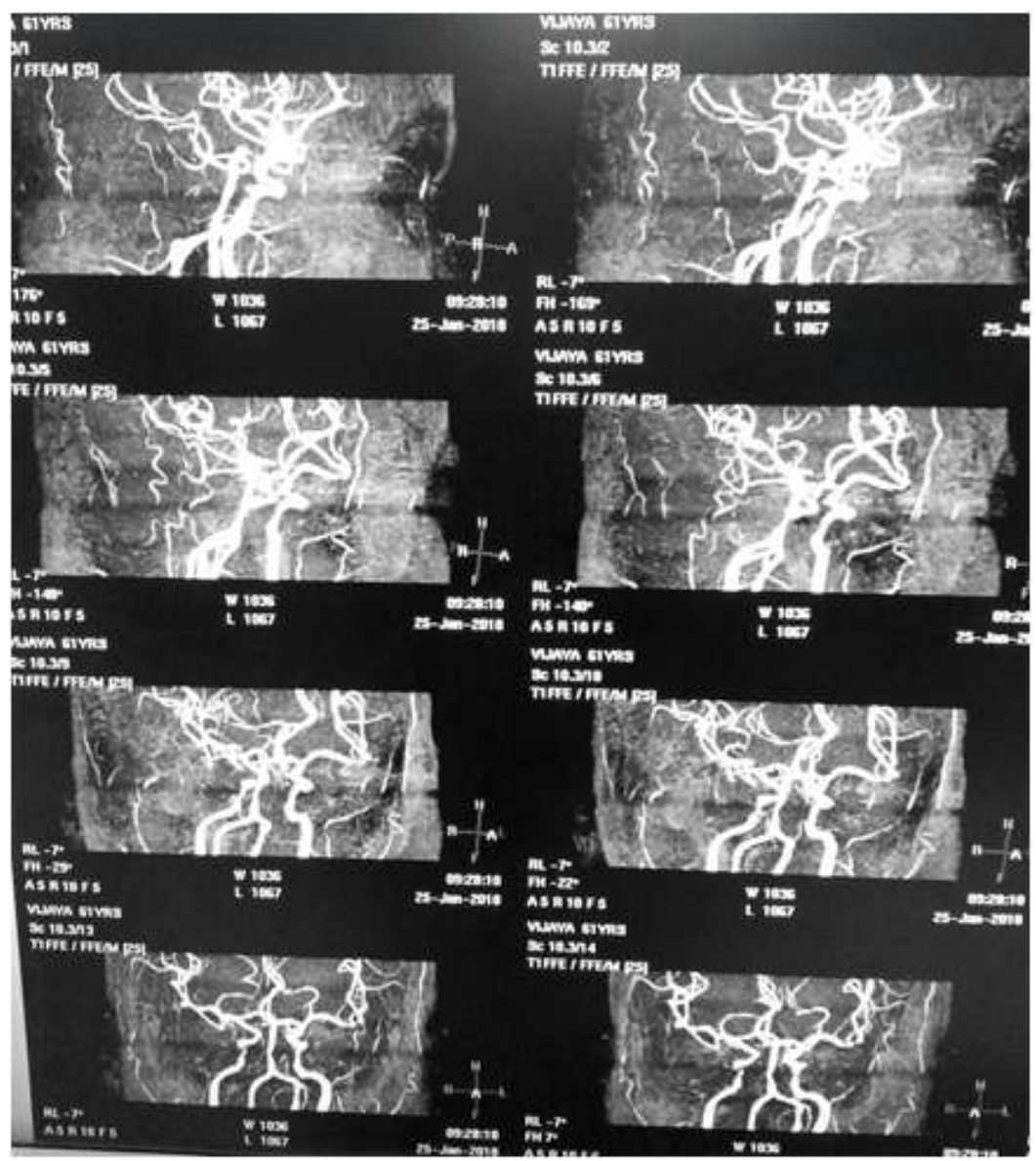

Fig. 8: MRA showing normal study

\section{Discussion}

In general, vascular supply of thalamus is carried out by multiple small vessels arising from posterior communicating arteries as well as P1 and P2 segments of posterior cerebral arteries. But there is significant and notable variations are seen. Regarding the vascular supply of thalamus, it is divided into four regions as follows: anterior, posterior, paramedian and inferolateral. Among these, blood supply of the paramedian territory is carried out by the thalamoperforating arteries arising from the P1 segment of the PCA. French neurologist Percheron described four variations in these thalamoperforating arteries. ${ }^{3}$ Out of these, AOP is an uncommon anatomic variant. This is a single arterial trunk arising from P1 segment of one of the two posterior cerebral arteries and branches from it supply bilateral thalami with varying contribution to rostral midbrain. Hence a characteristic pattern of ischemia involving bilateral paramedian thalamus with or without midbrain occurs when there is block in the flow of artery of Percheron. Features typical of bilateral thalamic infarcts are drowsiness, confusion, hypersomnolence, vertical gaze palsy, impaired memory, akinetic mutism and coma. ${ }^{4}$ If rostral midbrain is involved, patients will also have ophthalmoplegia, ataxia, hemiplegia and movement disorders. Our patient had most of the features of both thalamus and midbrain infarct as manifested by stuporous with confused state, vertical gaze palsy, oculomotor deficits like external ophthalmoplegia and ptosis as well as cerebellar ataxia.

Neuroimaging with MRI scan brain is the ideal modality for finding out the AOP infarct earlier. Especially the sequences like DWI and FLAIR $^{5}$ will demonstrate the thalamic paramedian infarction with or without involvement of the midbrain. A characteristic finding noted in MRI brain is the "V" sign that is a V- shaped hyperintensity seen on axial FLAIR and/or DWI sequences along the pial surface of the midbrain adjacent to the interpeduncular fossa. This is noticed in $67 \%$ of patients with midbrain involvement. ${ }^{6}$ Our patient's MRI brain demonstrated the $\mathrm{V}$ sign and the other characteristic MRI features. As AOP is very small, occlusion may not be visible in CT angiography or MR angiography and most of the time these angiographic studies are normal in these cases. ${ }^{7}$ In our patient also MR angiography could not demonstrate AOP occlusion.

Differential diagnoses for AOP occlusion producing bilateral thalamic infarction are deep cerebral venous thrombosis, Wernicke's encephalopathy, viral or demyelinating encephalitis, top of the basilar syndrome, extrapontine myelinolysis, bilateral thalamic glioma, Creutzfeldt-Jakob disease etc. ${ }^{8}$ Clinical features and imaging findings noted in our patient excluded these etiologies. 
Management of AOP infarction consists of thrombolysis if the patient present within the window period, intravenous heparin and long term antiplatelet therapy for secondary prevention. But in many occasions, thrombolysis is not done due to its narrow therapeutic window as well as the delay in making the diagnosis of AOP infarcts,. As our patient was brought to the hospital after the window period, thrombolysis could not be done. She was treated with heparin, antiplatelet, supportive care without ventilatory support.

Various studies report a mortality of about $12 \%$ after AOP infarction. ${ }^{9}$ Our patient, when she was discharged from hospital at 3 weeks, showed significant recovery in sensorium, ptosis, vertical gaze palsy and memory though there was some residual third nerve weakness on the right side with mild gait ataxia.

\section{Conclusion}

When a patient admitted in an intensive care unit with history of sudden onset of unconsciousness or altered sensorium, neurological causes are the common aetiology to be considered apart from many other causes. The neurological causes may be either structural (like trauma, stroke, SOL, etc.) or diffuse parenchymal (like infections, demyelination, metabolic, toxic, etc.). In a patient presents with clinical features of posterior circulation stroke, altered sensorium with behavioural changes and eye movement abnormalities are the clues to the diagnosis of AOP infarction. Neuroimaging with MRI brain especially the sequences like DWI and FLAIR are the best modality for making the diagnosis earlier. Treating physicians must be aware of the clinical and neuroimaging features of this rare stroke syndrome for the timely diagnosis and appropriate management.

\section{Acknowledgement}

We would like to thank Dr.N.Sundareswaran, Associate
Professor of Radiology of our institute for his valuable discussion regarding the radiodiagnosis of this patient.

\section{Conflict of Interest: Nil}

\section{References}

1. Kostanian V, Cramer SC. Artery of Percheron thrombolysis. Am J Neuroradiol 2007;28:870-1.

2. López-Serna R, González-Carmona P, López-Martínez M. Bilateral thalamic stroke due to occlusion of the artery of Percheron in a patient with patent foramen ovale: a case report. J Med Case Rep 2009;3:7392.

3. Percheron G. The anatomy of the arterial supply of the human thalamus and its use for the interpretation of the thalamic vascular pathology. Z Neurol 1973;205:1-13.

4. Arauz A, Patino-Rodriguez HM, Vargas-Gonzalez JC, Arguelles-Morales N, Silos H, Ruiz-Franco A et al, Clinical spectrum of artery of Percheron infarct: clinical-radiological correlations. J Stroke Cerebrovasc Dis 2014;23:1083-8.

5. Matheus MG, Castillo M. Imaging of acute bilateral paramedian thalamic and mesencephalic infarcts. AJNR Am J Neuroradiol 2003;24:2005-8.

6. Lazzaro NA, Wright B, Castillo M, Fischbein NJ, Glastonbury $\mathrm{CM}$, Hildenbrand PG, et al. Artery of Percheron infarction: imaging patterns and clinical spectrum. Am J Neuroradiol 2010;31(7):1283-9.

7. Sulena, Singh P, Kaur R, Kumar N. ischemic stroke due to occlusion of the artery of Percheron. Indian J Crit Care Med 2016;20:251-2

8. Vinod K.V, Kaaviya R, Arpita B. Artery of Percheron Infarction. Ann Neurosci 2016;23:124-6

9. Kulshreshtha D, Maurya PK, Chhirolya R, Singh AK, Thacker AK. Artery of percheron infarction: case reports and literature review. Int J Res Med Sci 2017;5:4629-31

How to cite this article: Gunasekaran K, Sivakumar S, Thiruvarutchelvan K. A case of rare stroke syndrome admitted in intensive care unit - Artery of Percheron infarction. India J Neurosci 2019;5(2):91-4. 